\title{
Particle Size Evolution during the Synthesis of Gold Nanoparticles Using In Situ Time-Resolved UV-Vis Spectroscopy: An Experimental and Theoretical Study Unravelling the Effect of Adsorbed Gold Precursor Species
}

Luca Panariello, Anand N. P. Radhakrishnan, Ioannis Papakonstantinou, Ivan P. Parkin, and Asterios Gavriilidis*
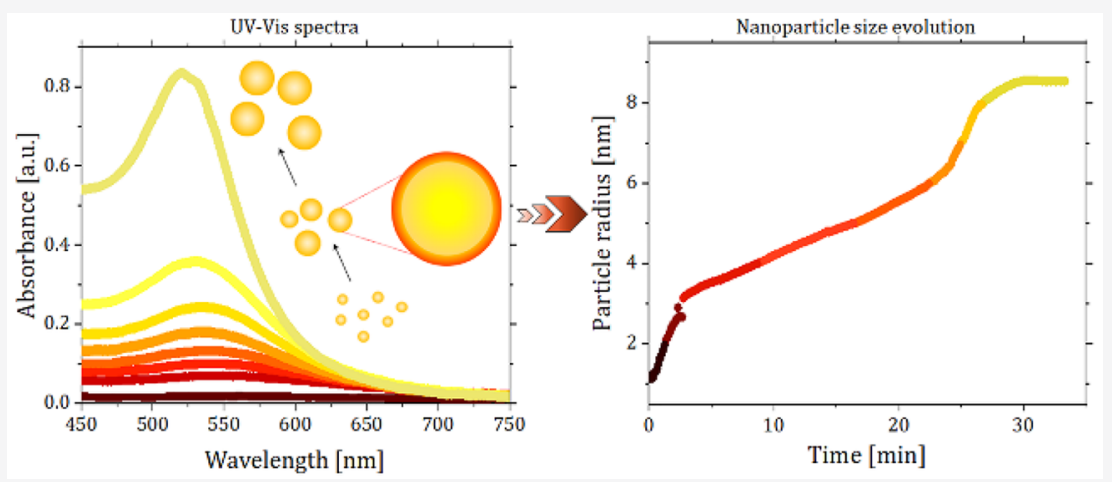

ABSTRACT: The quantitative analysis of nanomaterials synthesis kinetics is valuable both for understanding the synthesis and for development of manufacturing processes, and it usually requires the use of synchrotron-based instrumentation, making it challenging to perform experiments in the large parametric space needed to develop quantitative kinetic models. UV-vis spectroscopy represents a convenient technique to circumvent such difficulties, as it is available in most chemistry laboratories and allows fast data acquisition. This technique can in theory be used for the characterization of plasmonic nanomaterials synthesis kinetics. However, linking UV-vis spectra with characteristic features of the produced nanomaterials, such as size and shape, is a challenging task. This work presents a detailed spectroscopic analysis of gold nanoparticles syntheses via in situ time-resolved UV-vis spectroscopy, with emphasis on the role of gold precursor adsorption on the particle surface during nanoparticle growth. We show that the classic Turkevich synthesis and the growth of preformed gold nanoparticles with two different methods exhibit significant commonalities in the spectra evolution, explained in terms of the interaction between gold precursor species and the nanoparticle surface. Such interaction was accounted for in a model based on the Mie theory, describing the growing nanoparticles as core-shell spheres with an outer shell of few Ångströms characterized by reduced conductivity and increased electron damping rate. The proposed model led to the determination of the nanoparticle size and concentration evolution throughout the synthesis with good quantitative agreement against literature SAXS data. Furthermore, the core-shell model enabled the reproduction of the progressive blue-shift in the SPR peak position observed during the synthesis. Thus, this work reconciles the "seed-mediated growth" mechanism for the Turkevich synthesis with the temporal evolution of the spectra within the framework of the Mie theory, showing that the distinctive purplegreyish hue observed during the synthesis can be related to the interaction of gold precursor with the growing gold nanoparticles.

\section{INTRODUCTION}

Plasmonic nanoparticles represent an area of intense research due to their unique optical properties, making them attractive for a variety of applications in optics, electronics, biomedicine, etc. ${ }^{1,2}$ These nanoparticles are generally made of rare metals (e.g., gold or silver) or less expensive and more abundant materials, such as aluminum ${ }^{3}$ or magnesium, ${ }^{4}$ and exhibit absorbance peaks in the UV-vis-NIR region due to the collective oscillation of the electron cloud surrounding the core of their constitutive atoms. A plethora of different synthetic routes is available in the literature to obtain plasmonic 
nanoparticles of different material, ${ }^{5}$ shape, ${ }^{6}$ and assembly, ${ }^{7}$ in order to tailor their surface plasmon response to the desired application.

Among the plasmonic materials gold stands out, ${ }^{8}$ particularly in the field of biomedicine, ${ }^{9}$ in light of the proven biocompatibility of most gold nanostructures. ${ }^{10}$ The most common method for the synthesis of gold colloids is arguably the citrate reduction of chloroauric acid, also known as the Turkevich method. ${ }^{11}$ This synthesis consists of the reduction of gold precursor (chloroauric acid, $\mathrm{HAuCl}_{4}$ ) by trisodium citrate $\left(\mathrm{Na}_{3} \mathrm{C}_{6} \mathrm{H}_{5} \mathrm{O}_{7}\right)$ in water at a moderately high temperature $\left(\sim 100{ }^{\circ} \mathrm{C}\right)$. The outcome of the synthesis varies depending on the experimental conditions. Frens ${ }^{12}$ showed that by changing the ratio between $\mathrm{HAuCl}_{4}$ and citrate, the average particle size changes significantly. Ji et al. $^{13}$ demonstrated that this is due to different reaction mechanisms taking place when varying the gold-to-citrate ratio. These different mechanisms are a result of the $\mathrm{pH}$ variation due to the change of the $\left[\mathrm{HAuCl}_{4}\right] /[$ citrate $]$ ratio: $\mathrm{HAuCl}_{4}$ speciation is $\mathrm{pH}$ dependent, and the gold precursor reactivity decreases as the $\mathrm{pH}$ of the solution increases.

Since gold nanoparticles are plasmonic nanomaterials, UVvis spectroscopy is the simplest way to gather information regarding the mechanism of the reaction. This was extensively studied in the literature, ${ }^{13-16}$ nonetheless leading to somewhat unusual results. In particular, a progressive blue-shift of the spectra is observed during the reaction. This phenomenon has been attributed to an aggregative mechanism, with large aggregates forming in the early stage of the reaction that subsequently break down, leading to the formation of the final monodisperse product. ${ }^{16,17}$ These conclusions have been partially supported by ex situ TEM measurements, ${ }^{17,18}$ even though the applicability and accuracy of this technique for those cases are questionable, ${ }^{19}$ since drying artifacts and beaminduced particle nucleation/aggregation are common issues encountered when analyzing unstable specimens.

The aggregative mechanism was disproved by Polte and coworkers $^{20,21}$ for typical experimental conditions of the Turkevich synthesis, that is, for $\mathrm{HAuCl}_{4}$ concentration between 0.1 and $1 \mathrm{mM}$, [citrate $] /\left[\mathrm{HAuCl}_{4}\right]$ ratio between 5 and 20, and temperature between 75 and $100{ }^{\circ} \mathrm{C}$. For these conditions, the $\mathrm{pH}$ remains approximately constant throughout the reaction ${ }^{19}$ at a value between 5 and $7 .^{22}$ An extensive study of the synthesis at these conditions was carried out by Polte's group $^{19,20,22-24}$ through SAXS/XANES measurements with synchrotron radiation. This study led them to propose a "seedmediated growth" mechanism for this synthesis. ${ }^{19,20}$ According to this mechanism, gold nuclei are created from a supersaturated solution of $\mathrm{Au}(0)$ (nucleation step) and rapidly aggregate to form colloidally stable seeds. After this aggregation step, the seeds number remains constant over time, and the particles grow via surface reduction of the gold precursor. We recently reported a quantitative population balance model for the prediction of the particle size based on this mechanism, ${ }^{25}$ which accurately predicts the final particle size, whereas a previously available model not taking into account the decoupling between the aggregation and surface growth steps as well as the different reactivity of the various precursor species $^{26}$ failed in reproducing the synthesis outcome in several different conditions. ${ }^{27}$ However, this recent mechanistic description still leaves some unanswered questions, as apparently it is not in accordance with UV-vis spectroscopy results. The monotonic increase of the particle size observed via SAXS ${ }^{19,20}$ would suggest a progressive redshift in the SPR peak position during the synthesis, whereas experimental evidence shows a blue-shift in the SPR peak position from $\sim 550$ to $\sim 520 \mathrm{~nm}$. Regarding this discrepancy, Wuitschick et al. ${ }^{19}$ proposed that the reason for the spectra blue-shift during the synthesis stems from the interaction of the $\mathrm{HAuCl}_{4}$ molecules with the particle surface. A similar effect has been reported for the adsorption of $\mathrm{Ag}(\mathrm{CN})_{2}{ }^{-}$on the surface of preformed $\mathrm{Ag}$ nanoparticles ${ }^{28}$ and for the chelation of metal ions (at low concentration) on the surface of plasmonic particles. $^{29}$ In both cases, no aggregation was observed, but the spectra showed a higher peak absorption wavelength than expected due to ion adsorption.

This work addresses the apparent inconsistency between UV-vis spectroscopy results and the recent proposed reaction mechanism for the Turkevich synthesis by fitting in situ timeresolved UV-vis spectra recorded throughout the synthesis with a model based on the Mie theory. The proposed model takes into account the presence of adsorbed gold precursor on the particle surface by considering the growing particle as a core-shell structure, with a thin outer shell representing the portion of the particle interacting with the adsorbed $\mathrm{Au}$ precursor species. A similar model was employed by Peng et al. ${ }^{30}$ to explain the nonmonotonic trend of the SPR position of silver nanoparticles for decreasing particle size, and by Averitt et al. $^{31}$ to model Au-coated $\mathrm{Au}_{2} \mathrm{~S}$ nanostructures (though in this case a dielectric core represented the $\mathrm{Au}_{2} \mathrm{~S}$ cluster, while the outer shell described the Au coating). We show that the simple model proposed in this work can reproduce the evolution of the two main spectra characteristics during the synthesis: the SPR peak absorbance increase and the progressive shift toward higher energies of the SPR peak as the reaction time increases. To further support our hypotheses, we examined the growth of preformed gold nanoparticles (with no further nucleation occurring) with two different methods, namely citrate reduction of $\mathrm{HAuCl}_{4}$, i.e., "citrate growth", ${ }^{23}$ and "hydroxylamine growth" of gold nanoparticles. ${ }^{32}$ The same behavior in the SPR position is observed, with a significant redshift at the beginning of the reaction (upon injection of the gold precursor) and a progressive blue-shift in the SPR peak position, further suggesting the validity of our model.

The manuscript is organized as follows: first, the details of the experimental procedure used in the work are described; then, in the first subsection of the Results and Discussion, we summarize and discuss the model employed to fit the data. We then comment on the experimental results, with emphasis on the distinctive features observed in the spectra, as well as the aspects that bridge our experimental results with SAXS measurements from the literature. We next compare the results obtained from fitting the spectra with SAXS measurements from the literature. The model is subsequently used to study particle evolution during seeded growth. Eventually, the conclusions of the work are presented in the final section of the manuscript.

\section{EXPERIMENTAL SECTION}

Materials. Gold(III) chloride trihydrate $\left(\mathrm{HAuCl}_{4} \cdot 3 \mathrm{H}_{2} \mathrm{O}, \geq\right.$ 99.9\%) and citric acid monohydrate (ACS reagent grade $\geq 99.0 \%$ ) were purchased from Sigma-Aldrich. Trisodium citrate dihydrate (99\%) was purchased from Alfa-Aesar. Hydroxylamine hydrochloride (+99.0\%) was purchased from Acros Organics. 
Synthesis and Characterization of Gold Nanoparticles. All reactions were carried out in a quartz cuvette (Hellma Optics) with $10 \mathrm{~mm}$ optical path placed in a UV-vis cuvette holder equipped with Peltier elements to allow for temperature control (Qpod, Quantum Northwest). The liquid in the cuvette was kept under agitation with the aid of a magnetic stirrer (stirring speed $3000 \mathrm{rpm}$ ). The liquid volume employed in each synthesis was $1.5 \mathrm{~mL}$. TEM micrographs of the particles were obtained with a JEOL 1200 EX microscope with a tungsten filament operating at an acceleration voltage of $120 \mathrm{kV}$. TEM samples were prepared by casting $\sim 5 \mu \mathrm{L}$ of colloidal solution on a carbon coated copper grid. Particle size distributions from the TEM images were obtained using the software Pebbles. ${ }^{33}$

Gold seeds ( $8.9 \mathrm{~nm}$ radius) were synthesized according to the Turkevich method, ${ }^{11}$ replicating the protocol from Polte et al. $^{20}$ In detail, for given final concentrations of $0.25 \mathrm{mM}$ of $\mathrm{HAuCl}_{4}$ and $2.5 \mathrm{mM}$ of trisodium citrate, $75 \mu \mathrm{L}$ of a $5 \mathrm{mM}$ stock solution of $\mathrm{HAuCl}_{4}$ was added to $1.35 \mathrm{~mL}$ of preheated DI water (at a temperature of $85^{\circ} \mathrm{C}$ ), followed after $\sim 3 \mathrm{~min}$ by the addition of $75 \mu \mathrm{L}$ of a $50 \mathrm{mM}$ solution of trisodium citrate, separately preheated to the reaction temperature.

In the "citrate growth" experiment, we replicated the protocol from Polte et al. ${ }^{23}$ In detail, after completing the synthesis of the seeds ( $8.9 \mathrm{~nm}$ radius) via the above-mentioned Turkevich procedure $\left(\left[\mathrm{HAuCl}_{4}\right]=0.25 \mathrm{mM}\right.$, [citrate $]=2.5$ $\mathrm{mM}, 85^{\circ} \mathrm{C}$ ), the obtained particles were kept at a temperature of $85^{\circ} \mathrm{C}$. After the seeds synthesis terminated (that is, after the $\mathrm{UV}$-vis spectra stabilized, $\sim 40 \mathrm{~min}$ ), $19 \mu \mathrm{L}$ of $5 \mathrm{mM} \mathrm{HAuCl}_{4}$ was added to the solution. After the addition of the fresh precursor, the $\mathrm{Au}$ (III) concentration was equal to $0.0625 \mathrm{mM}$.

The "hydroxylamine growth" experiment was performed starting from $6.3 \mathrm{~nm}$ radius Au seeds prepared according to Panariello et al. ${ }^{34}$ In brief, a $0.2 \mathrm{mM} \mathrm{Au}(\mathrm{III})$ solution (initial $\mathrm{pH}=12$, adjusted with $\mathrm{NaOH} 2 \mathrm{M}$ ) was preheated to $90{ }^{\circ} \mathrm{C}$. Subsequently, concentrated citric acid $(72 \mathrm{mM})$ was added to the solution (citric acid:Au(III) 12:1 mol:mol after addition). The final $\mathrm{pH}$ of the seeds solution (measured at room temperature) was approximately 5.6. After synthesis, the seeds were diluted to yield a final $\mathrm{Au}(0)$ concentration of $0.03 \mathrm{mM}$. Then, particle growth was performed at room temperature according to the protocol proposed by Brown and Natan with minor changes: ${ }^{32} 7 \mu \mathrm{L}$ of a $0.2 \mathrm{M}$ hydroxylamine hydrochloride solution was added to $1.487 \mathrm{~mL}$ of the diluted $6.3 \mathrm{~nm}$ radius gold nanoparticles solution. After $1 \mathrm{~min}, 6 \mu \mathrm{L}$ of a 25 $\mathrm{mM} \mathrm{Au}(\mathrm{III})$ solution was added and stirring of the solution was continued for $10 \mathrm{~min}$.

\section{RESULTS AND DISCUSSION}

Spectra Modeling Framework. Following the experimental evidence gathered from Polte's group via SAXS, ${ }^{19,20}$ we modeled the growing particles as single spherical entities. However, to account for the adsorption of gold precursor on the particle surface, the spherical gold nanoparticle was described as a core-shell structure embedded in a solvent of dielectric constant $\varepsilon_{m}$ (see Figure 1), following the same framework employed by Peng et al. ${ }^{30}$ to describe the optical properties of silver nanoparticles. The detailed model is reported in the Supporting Information, Section S1. In the following, all the quantities in the model eqs S.1-S.16 referring to the core have the subscript $c$ (e.g., $A_{c}^{*}$ ), while they have the subscript $s$ when referring to the shell (e.g., $A_{s}^{*}$ ).

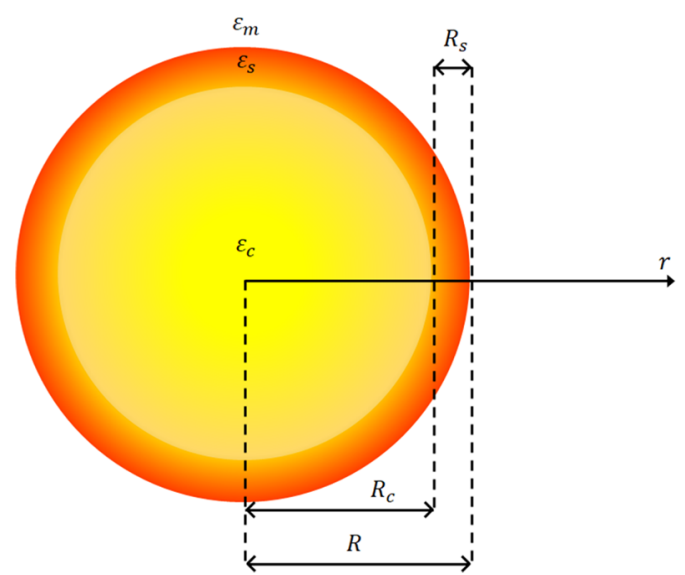

Figure 1. Modeling domain used in this work. The inner core of radius $R_{c}$ and dielectric constant $\varepsilon_{c}$ represents the fraction of gold nanoparticle not directly affected by surface phenomena. The outer shell of thickness $R_{s}$ surrounding the particle represents the region of interaction between the $\mathrm{Au}$ precursor species and the particle, characterized by a dielectric constant $\varepsilon_{s}$. The particle is surrounded by a nonabsorbing solvent of dielectric constant $\varepsilon_{m}$ (in this specific work, water).

The parameters determining the value of the dielectric constants of core and shell are the core and shell radius $\left(R_{c}\right.$ and $\left.R_{s}\right)$, the core and shell electron density scale factor $\left(g_{c}\right.$ and $\left.g_{s}\right)$ and conduction electron damping frequency correction factor $\left(A_{c}^{*}\right.$ and $\left.A_{S}^{*}\right)$. Following the work of Mulvaney et al. ${ }^{35}$ and Peng et al., ${ }^{30,36}$ we introduced the parameter $g$ to account for changes in the particle free electron density due to the interaction of the particle with the precursor species (see eq S.15), while the parameter $A^{*}$ corrects the conduction electron damping frequency, accounting for nonlocal effects arising particularly in particles with radii smaller than $5 \mathrm{~nm} .{ }^{37}$ The inner core of the nanoparticle represents the largest fraction of the sphere, with radius $R_{c}$ and dielectric constant $\varepsilon_{c}\left(R_{c}, \omega\right)$ (with $\omega$ radiation frequency), described according to eqs S.12-S.16. Assuming that the particle core is not involved in interactions with metal precursor species present in solution, we set $g_{c}=1$ in eq $\mathrm{S} .15$ and $A_{c}^{*}=1$ in eq $\mathrm{S} .16$ (value theoretically predicted for a sphere ${ }^{38}$ ). The particle core is surrounded by a thin shell of thickness $R_{s}$ and dielectric constant $\varepsilon_{s}$, representing the region where the gold precursor species attach to the gold nanoparticle surface. $\varepsilon_{s}$ is also given by eqs S.12-S.16, however, accounting for the following points:

- Changes in the free electron density due to adsorption of gold precursor species. Therefore, we hypothesize that the scaling factor $g_{s}$ in eq $S .15$ is smaller than 1 , representing a decrease in the free electron density $N_{q, s}=$ $g_{s} N_{q}$ caused by the adsorption of gold precursor species on the surface of the particles. ${ }^{28,30,35,36,39}$

- Possible spectra damping due to adsorption of the precursor on the particle surface. We subsequently treat the parameter $A_{s}^{*}$ in eq $S .16$ as a fitting parameter.

The value of $R_{s}$ was also set as a fitting quantity, expected to be in the order of few Ångströms, as the shell should be composed only of a small number of atomic layers. A similar approach has been adopted by Peng et al. ${ }^{30}$ to explain the nonmonotonic changes of the SPR peak position for silver nanoparticles, whose radius ranged between 1 and $9 \mathrm{~nm}$. Since the particles studied in this work are below $15 \mathrm{~nm}$ in radius, we 
use the dipole approximation to model them by applying eqs S.2 and S.9-S.11 to calculate the extinction coefficient of the particles. ${ }^{15,37,40}$ Equations S.12-S.16 are used to model the dielectric properties of the particles. The unknown quantities in these equations are then $R_{c}, R_{s}, N, g_{s}$, and $A_{s}^{*}$. The other parameters appearing in the model and various constants in eqs S.1, S.2, and S.9-S.16 are reported in Table S1.

Each experimental spectrum was fitted with the aid of the commercial software Matlab, returning the parameters used in the model to describe the spectrum $\left(R_{c}, R_{s}, N, g_{s}, A_{s}^{*}\right)$. Details of the fitting procedure are provided in the Supporting Information, Section S2. In brief, each set of spectra obtained from an experiment was fitted starting from a time point where the particle features were known via ex situ TEM measurements (e.g., the end of the synthesis for the seedless Turkevich synthesis or beginning of the synthesis for the seeded-growth syntheses). The ex situ determined particle features were used as initial guess for the first spectra fitting according to eqs S.1, S.2, and S.9-S.16. The results obtained from the ith spectrum fitting (at time, $t=t_{i}$ ) were used as initial guess for the fitting of the next spectrum (see Supporting Information, Section S2).

Synthesis of Gold Nanoparticles via the Turkevich Method. The absorption at the SPR peak $\left(\mathrm{Abs}_{\mathrm{SPR}}\right)$ is proportional to the product between the particle number density $N$ and the extinction coefficient at the SPR peak wavelength $\sigma_{\text {ext }}\left(\lambda_{\mathrm{SPR}}\right)$ (see eq S.1). The latter is proportional to the third power of the particle radius $R^{40,41}$ (eq S.8). Figure 2a shows the cubic root of the $\mathrm{Abs}_{\mathrm{SPR}}$ as a function of time. Note that the $\sqrt[3]{\mathrm{Abs}_{\mathrm{SPR}}}$ is proportional to the product $\sqrt[3]{N} R$. One can clearly distinguish three regions in the graph:

I. An initial region where $\sqrt[3]{\mathrm{Abs}_{\mathrm{SPR}}}$ increases in a superlinear fashion;

II. An intermediate region where $\sqrt[3]{\mathrm{Abs}_{\mathrm{SPR}}}$ increases linearly;

III. A final region where $\sqrt[3]{\mathrm{Abs}_{\mathrm{SPR}}}$ exponentially increases and levels off to its final value.

The same three regions were identified by Polte et al..$^{20}$ via in situ time-resolved SAXS measurements (Figure 2b). The authors assigned to each region a different reaction stage, which can be also observed in the $\sqrt[3]{\mathrm{Abs}_{\mathrm{SPR}}}$ curve:

I. A first stage where the particles grow via aggregation: here the number density of the particles decreases, while the particle size increases (region I of the $\sqrt[3]{\mathrm{Abs}_{\mathrm{SPR}}}$ curve);

II. A second stage where no further aggregation occurs, and the particles grow linearly in time via a surface growth reaction; the same linear trend is observed in region II of the $\sqrt[3]{\mathrm{Abs}_{\mathrm{SPR}}}$;

III. A third final stage, where the particle size grows via an autocatalytic growth stage and levels up to its final value, equivalent to the region III of the $\sqrt[3]{\mathrm{Abs}_{\mathrm{SPR}}}$ curve.

The behavior of the SPR peak position $\left(\lambda_{\text {SPR }}\right)$ apparently contradicts the "seed-mediated growth" mechanism: $\lambda_{\mathrm{SPR}}$ moves from $\sim 550 \mathrm{~nm}$ to $\sim 520 \mathrm{~nm}$ during the synthesis (Figure $2 \mathrm{a}$ ). The trend observed in $\lambda_{\mathrm{SPR}}$ during the reaction was explained in the past with the formation of large aggregates in the first part of the synthesis, which subsequently break down leading to the final monodisperse product. ${ }^{16,17}$ However, Polte et al. ${ }^{20}$ demonstrated via in situ time-resolved ultrasmall angle X-ray
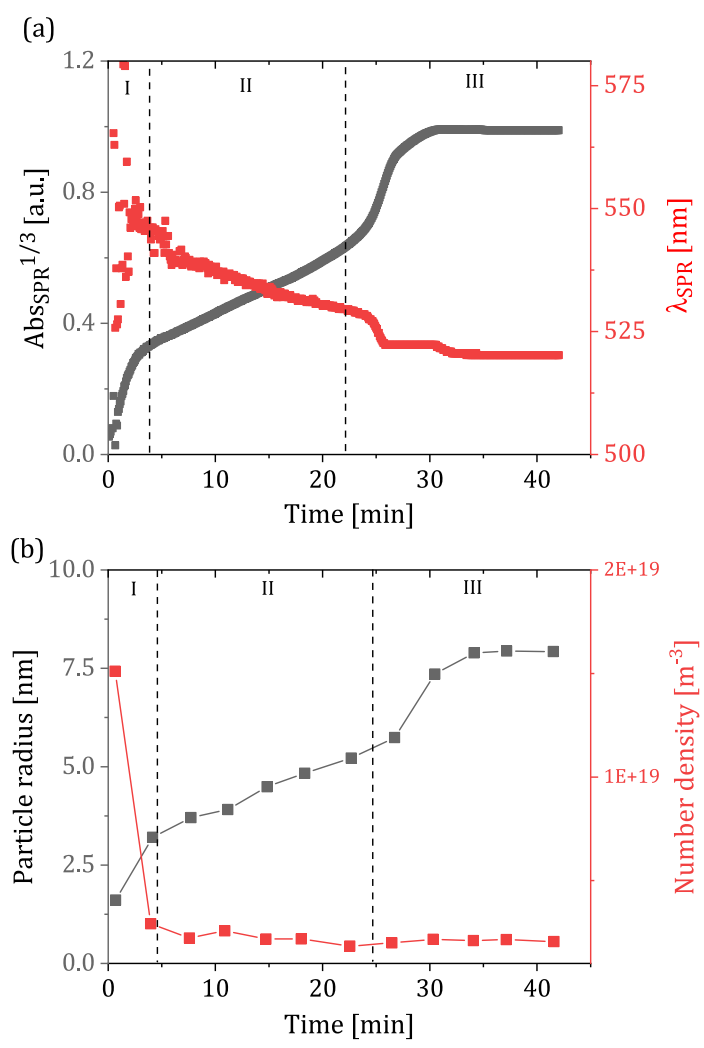

Figure 2. (a) $\sqrt[3]{\mathrm{Abs}_{\mathrm{SPR}}}$ and SPR peak position $\lambda_{\mathrm{SPR}}$ versus time, obtained via in situ time-resolved UV-vis spectroscopy during the Turkevich synthesis of gold nanoparticles performed in this work $\left(\left[\mathrm{HAuCl}_{4}\right]=0.25 \mathrm{mM},[\right.$ citrate $]=2.5 \mathrm{mM}$ and temperature of 85 $\left.{ }^{\circ} \mathrm{C}\right)$. (b) Evolution of particle radius and number density from SAXS measurements performed by Polte et al. ${ }^{20}$ during the same synthesis. Three distinct regions (I-III) can be observed in both graphs. Each region corresponds to a different reaction stage: (I) nanoparticle nucleation/aggregation, (II) slow linear particle growth, and (III) fast final particle growth.

scattering (USAXS) that no large aggregates are present at any time during the reaction. The progressive blue-shift of the peak position from 550 to $520 \mathrm{~nm}$ could then be caused by the adsorption of $\mathrm{Au}$ precursor species on the surface of the particles. As a matter of fact, Biggs et al. ${ }^{42}$ observed via AFM measurements the adsorption of $\mathrm{Au}(\mathrm{III})$ species on the surface of citrate coated gold nanoparticles upon addition of $\mathrm{HAuCl}_{4}$ to the colloidal solution. The interaction of $\mathrm{Au}$ precursor species with the gold particle surface is also supported by the results from Polte et al., ${ }^{23}$ who studied the citrate reduction of $\mathrm{HAuCl}_{4}$ on the surface of preformed gold nanoparticles. They noticed that upon injection of the gold precursor in the seeds solution at room temperature (hence, with no reduction of the precursor taking place), the SPR peak shifted to a higher wavelength, the SPR intensity decreased and the peak width increased; however, the SAXS spectra of the particles before and after addition of the precursor appeared identical, suggesting that the changes in the spectra are linked to surface phenomena rather than aggregation of the particles (see Supporting Information, Section S3). Similar observations are reported by Hendel et al., ${ }^{43}$ who studied the relation between UV-vis spectra and gold nanoparticle concentration; when diluting $\mathrm{Au}$ nanoparticle solutions with $\mathrm{HAuCl}_{4}$ solutions, the authors observed a systematic red-shift and increase in the SPR peak width, even though no reaction was taking place. The 
literature discussed led us to hypothesize that, during the initial stage of the reaction, the gold precursor species bind to the particle surface, causing the SPR peak to be initially located at $\sim 550 \mathrm{~nm}$. During the synthesis, as the gold precursor adsorbs on the particle surface and is progressively consumed, $\lambda_{\mathrm{SPR}}$ shifts from 550 to $520 \mathrm{~nm}$.

The synthesis investigated led to particles with an average radius of $8.9 \mathrm{~nm}$ (Supporting Information, Figure S2a) with a reaction time of $30 \mathrm{~min}$, values very close to the experiments performed by Polte et al., ${ }^{20}$ who obtained particles with an average radius of $8 \mathrm{~nm}$ with a reaction time of $35 \mathrm{~min}$. The discrepancies can be attributed to experimental error, as well as inherent lack of complete reproducibility of the standard Turkevich synthesis, as reported by Wuithschick et al. ${ }^{19}$ In their work the authors showed that different researchers performing the standard Turkevich synthesis at $100{ }^{\circ} \mathrm{C}$ obtained particles with radii ranging from $\sim 6.5$ to $\sim 11 \mathrm{~nm}$.

To explain the optical behavior observed during the synthesis, we modeled the gold nanoparticles as a core-shell structure, as described in the previous section and in the Supporting Information, Section S1. The particle core has no direct interaction with the $\mathrm{Au}$ precursor species; on the other hand, the strong binding of the gold precursor species with the particle surface needs to be accounted for a proper description of the spectra. Therefore, we modeled the outer part of the sphere as a shell with damping frequency correction factor $A_{s}^{*}$ $>1$ and free electron density scale factor $g_{s}<1$.

Therefore, to compute the spectra at each reaction time $t$ through eqs S.1, S.2, and S.9-S.16, five parameters need to be fitted: the core size $R_{c}$, the shell size $R_{s}$, the shell parameters $A_{s}^{*}$ and $g_{s}$ and the particle number density $N$. The results of the fitting in terms of particle radius, number density and volume fraction are reported in Figure $3 \mathrm{a}-\mathrm{c}$. Here, one can observe the good agreement with the data reported from Polte et al., ${ }^{20}$ with the discrepancy observed likely due to the inherent lack of perfect reproducibility when performing the experiment (possibly due to different reactor scale and configuration, gold precursor stock, etc.).

When comparing the fitting results with the SAXS measurements from Polte et al. $^{20}$ (Figure $3 a-c$ ), we can observe in both data sets the same three reaction stages (i.e., nucleation/aggregation, linear growth, and autocatalytic growth). It is worth stressing that the $\mathrm{pH}$ of the final colloidal solution was approximately 6.2 , which is larger than the $\mathrm{pH}$ value of 5.6 required for optimal reproducibility of the synthesis. $^{22}$ Considering the discussed inherent lack of reproducibility, the agreement between the SAXS and UVvis determined radii, number densities and volumetric fractions is satisfactory. Despite the model simplicity, the fitting quality of the full spectra is acceptable throughout the synthesis (see Figure S3a,b), with good estimation of the SPR peak absorbance and position compared to those determined experimentally (see Figure 4a,b).

During the synthesis, the fitting returned a constant outer shell thickness of $0.5 \mathrm{~nm}$, which started to slowly decrease after $\sim 25 \mathrm{~min}$, reaching zero when the synthesis was over (Figure $5 a)$. The free electron density of the shell decreased during the synthesis by a factor $g_{s} \sim 0.85$, down to $\sim 5.1 \times 10^{28} \mathrm{~m}^{-3}$ (Figure $5 \mathrm{~b}$ ). The correction factor of the conduction electron damping frequency $A_{s}^{*}$ initially increased from 1 to 1.5 and then decreased to 1 after approximately $15 \mathrm{~min}$ (Figure 5c), when the particle radius was $\sim 5 \mathrm{~nm}$. Once the growth process

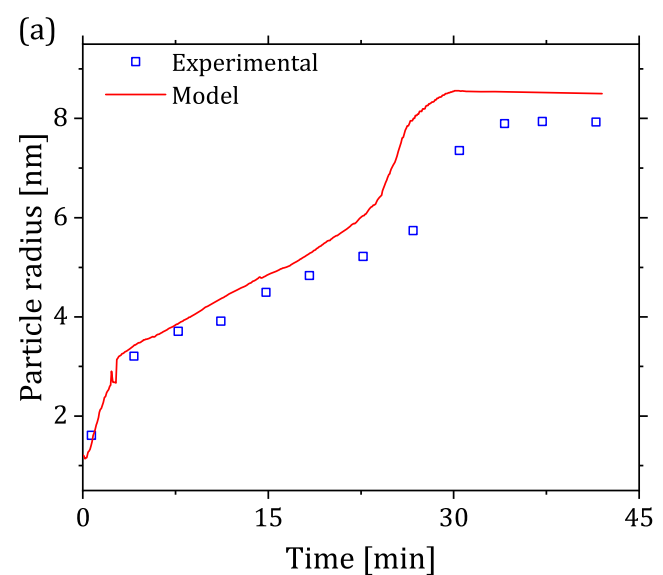

(b)

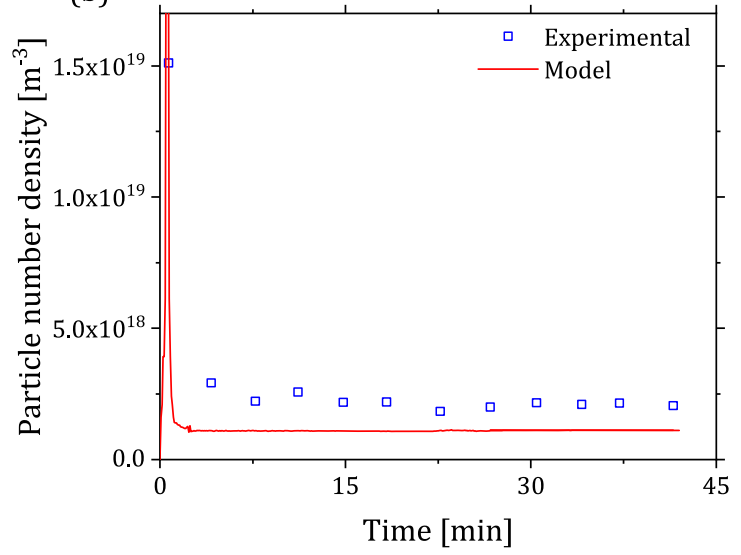

(c)

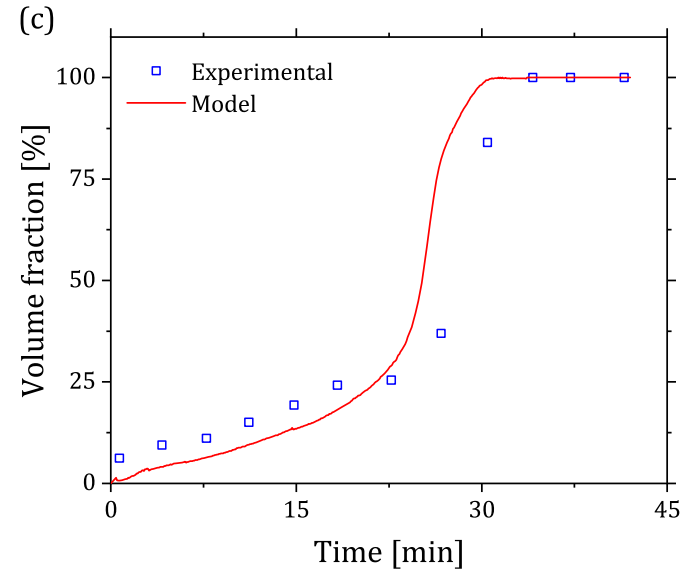

Figure 3. (a) Particle radius, (b) particle number density, and (c) volume fraction (given by $N(t) R(t)^{3} / N_{\text {final }} R_{\text {final }}{ }^{3}$ ) obtained from the fitting of the UV-vis data from the experiments performed in this work (solid red lines) and comparison with the SAXS experimental data obtained from Polte et al. ${ }^{20}$ for the same Turkevich synthesis (blue open symbols); experiments conducted with $\left[\mathrm{HAuCl}_{4}\right]=0.25$ $\mathrm{mM}$, [citrate] $=2.5 \mathrm{mM}$, and temperature of $85{ }^{\circ} \mathrm{C}$.

terminated, the shell thickness decreased to 0 and the free electron density increased to the bulk gold value.

The trends reported in Figure $5 a-c$ can be justified from a physical chemistry point of view considering the possible effects induced by the adsorption of the Au precursor species on the surface of the nanoparticles. The reduction of $\mathrm{Au}$ precursor to $\mathrm{Au}(0)$ during the Turkevich synthesis can be described according to the following two reactions: ${ }^{25,44,45}$ 

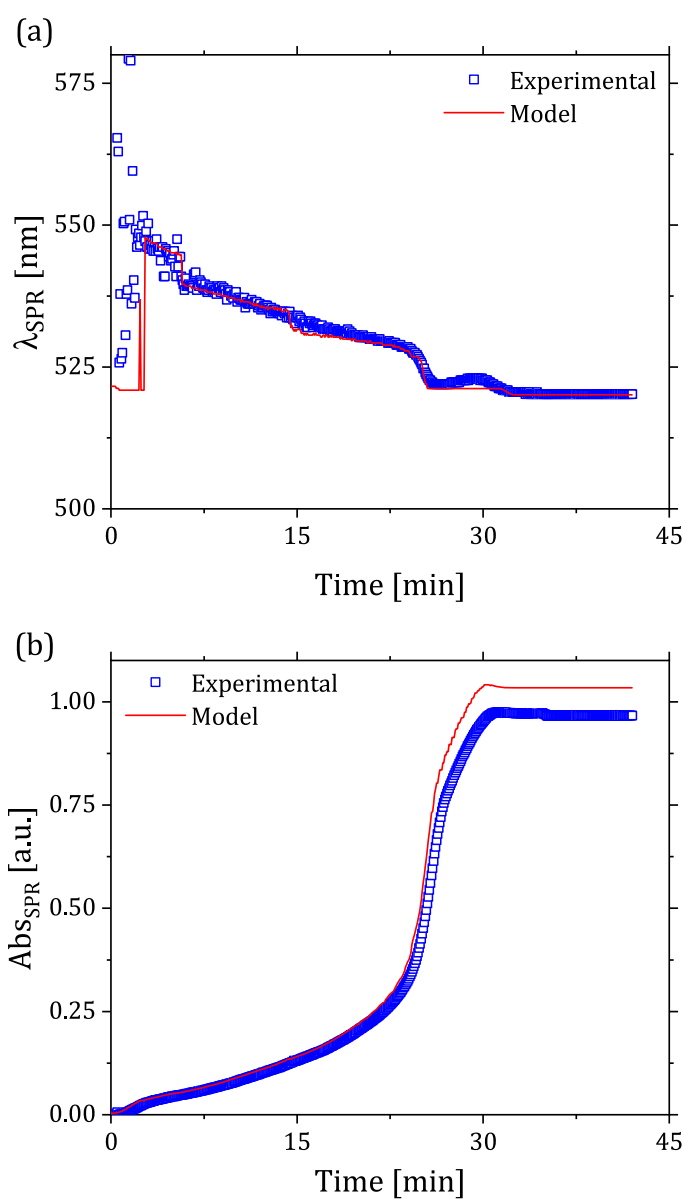

Figure 4. Modeled (solid red line) and experimentally measured (open blue symbols) (a) SPR peak position, $\lambda_{\mathrm{SPR}}$ and (b) peak absorbance, $\mathrm{Abs}_{\mathrm{SPR}}$, throughout the Turkevich synthesis. Experimental data acquired in this work. Experimental conditions: $\left[\mathrm{HAuCl}_{4}\right]=0.25$ $\mathrm{mM}$, [citrate] $=2.5 \mathrm{mM}$, and temperature of $85^{\circ} \mathrm{C}$.

$$
\begin{aligned}
& \mathrm{AuCl}_{4}^{-}+\mathrm{C}_{6} \mathrm{H}_{5} \mathrm{O}_{7}^{3-} \\
& \quad \rightarrow \mathrm{C}_{5} \mathrm{H}_{4} \mathrm{O}_{5}{ }^{2-}+\mathrm{AuCl}_{2}{ }^{-}+\mathrm{CO}_{2}+2 \mathrm{Cl}^{-}+\mathrm{H}^{+} \\
& 3 \mathrm{Au}(\mathrm{I}) \rightarrow 2 \mathrm{Au}(0)+\mathrm{Au}(\mathrm{III})
\end{aligned}
$$

The $\mathrm{Au}(\mathrm{III})$ precursor is present in the solution throughout the whole synthesis, as observed by Polte et al. via XANES measurements, ${ }^{20}$ with the oxidation state of gold in solution during the synthesis being above 1 for approximately the whole duration of the process. As already discussed, Au precursor species have more affinity to the Au nanoparticle surface than citrate molecules. ${ }^{42}$ Due to this affinity, we hypothesize that the initially formed Au particles adsorb Au precursor species on their surface. Both $\mathrm{Au}(\mathrm{I})$ and $\mathrm{Au}(\mathrm{III})$ species can be involved in this process, due to their likely coexistence during the synthesis. While the interaction between gold precursor and the gold nanoparticle surface is supported by experimental evidence as discussed earlier in the text, ${ }^{23,42,43}$ the mechanism of this process is not yet clear. The adsorption of the $\mathrm{Au}$ precursor species on the surface of the particles can induce a decrease in the free electron density of the particle surface; then, the reducing agent (citrate) provides the electrons required for the reduction of the adsorbed precursor. Once the $\mathrm{Au}$ precursor species have been converted to $\mathrm{Au}(0)$, other precursor present in the solution bulk can adsorb on the
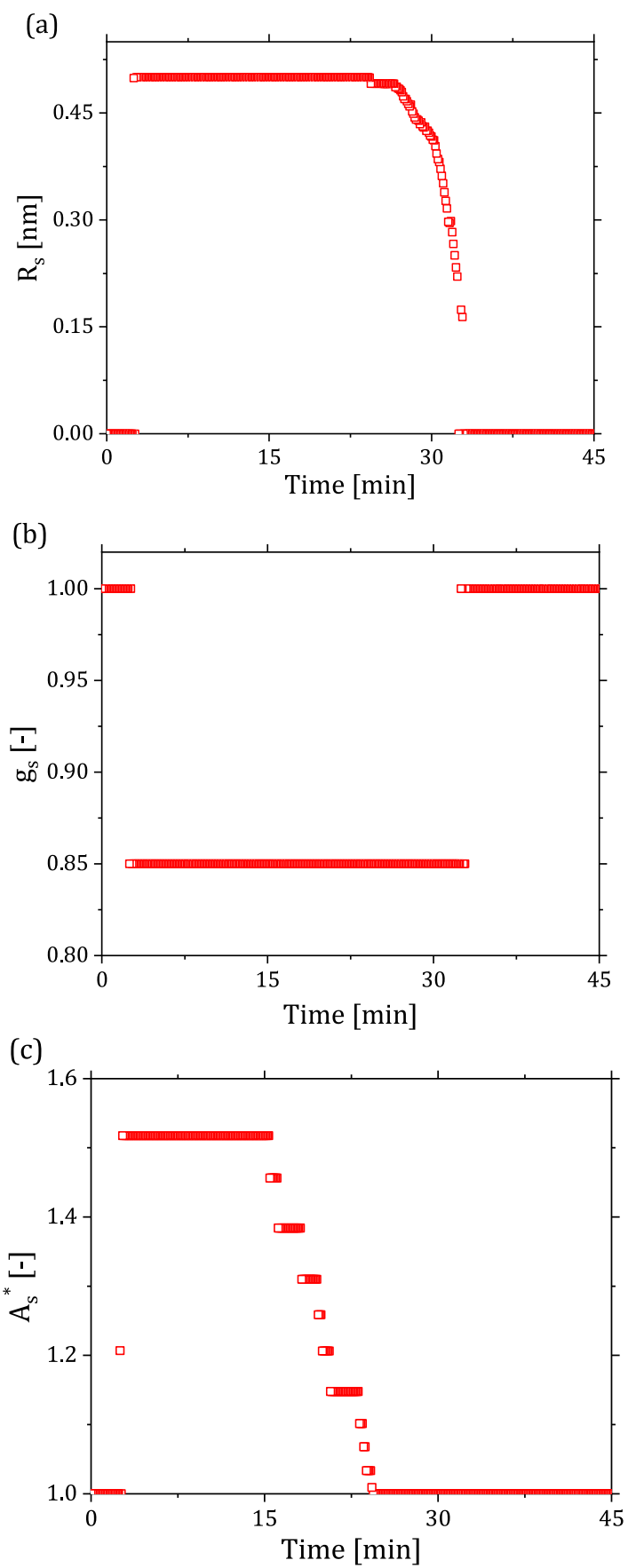

Figure 5. Evolution of (a) outer shell thickness $R_{s}$, (b) electron density scale factor $g_{s}$, and (c) conduction electron damping frequency correction factor $A_{s}^{*}$, during the Turkevich synthesis. Experimental conditions: $\left[\mathrm{HAuCl}_{4}\right]=0.25 \mathrm{mM}$, [citrate] $=2.5 \mathrm{mM}$, and temperature of $85^{\circ} \mathrm{C}$.

particle surface, with this cycle repeating itself until full precursor conversion. A similar mechanism was observed by Mulvaney et al., ${ }^{28}$ where the authors studied a seeded-growth experiment of $\mathrm{Ag}$ nanoparticles. The addition of $\mathrm{Ag}(\mathrm{CN})_{2}{ }^{-}$to preformed silver seeds caused a red-shift of the spectra in absence of any reducing agent. Then, radiolytically generated reducing organic radicals caused the reduction of the adsorbed silver ions and subsequent particle growth. After the radical generation, the spectra blue-shifted to the initial position, with an increase in absorbance and peak sharpening due to particle growth. The authors inferred that the adsorption of the 
precursor on the preformed seeds caused a decrease in the free electron density, leading to the spectra red-shift. Once the reducing radicals were introduced in the system, these compounds would inject electrons in the particles, which would then be used to reduce the adsorbed precursor, and the SPR peak would subsequently blue-shift and increase in intensity. The inverse phenomenon has been observed by Novo et al., ${ }^{46}$ who monitored the SPR evolution during the gold-catalyzed oxidation of ascorbic acid. The spectra of the gold catalyst blue-shifted upon addition of ascorbic acid due to the injection of electrons from the compound being oxidized (ascorbic acid) to the surface of the gold nanoparticles. At the end of the process, the spectra of the catalyst shifted back to the initial position as the oxidizing agent (in this case oxygen) removed the electrons transferred from ascorbic acid to the catalyst.

Chemisorption is also reported to cause a faster coherence loss of the free electrons due to the introduction of new electrons relaxation pathways. ${ }^{40,41,47}$ This phenomenon translates in an increase in the damping frequency $\gamma_{d}$, which is observed in our fitting results in the increase of $A_{s}^{*}$ in the first $15 \mathrm{~min}$ of the synthesis (Figure 5c). One can note from Figure $5 \mathrm{a}-\mathrm{c}$ that, after approximately $15 \mathrm{~min}, A_{s}^{*}$ abruptly decreases from 1.5 to 1 . The shell thickness instead starts decreasing from the value of $0.5 \mathrm{~nm}$ only after $\sim 25 \mathrm{~min}$, leveling off at 0 after $\sim 30 \mathrm{~min}$, when the reaction is over. Similarly, $g_{s}$ remains constant and equal to 0.85 until the reaction is completed. These three trends suggest that the damping frequency of the outer shell electrons $\gamma_{d, s}$ is mainly affected in the first $15 \mathrm{~min}$ of the reaction, possibly due to the smaller particle size (below 5 $\mathrm{nm}$ in radius), whereas the free electron density variations take place during the whole synthesis. This is not surprising, as small particles are more sensitive to changes in the surface chemistry than larger ones, as observed from the effect of thiols coating on Au nanoparticles of different sizes. The coating of particles smaller than $5 \mathrm{~nm}$ with thiols is reported to cause SPR peak damping (that is, an increase in $\gamma_{d}$ ), whereas particles larger than $5 \mathrm{~nm}$ coated with thiols only show red-shift of the spectra. $^{41}$ Finally, one should also consider that citrate molecules compete with the Au precursor for adsorption on the particle surface. Since citrate is in excess, it is not possible to rule out the fact that citrate could be present on the particle surface together with the gold precursor, causing an inhomogeneous coating on the particles, possibly contributing to the evolution of both $A_{s}^{*}$ and $g_{s}$.

Seeded-Growth Syntheses. We then considered the possibility that the effect of the Au precursor adsorption on the particle surface manifested in other syntheses. We therefore performed a seeded-growth experiment ("citrate growth") reproducing Polte et al., ${ }^{23}$ who conducted the same synthesis examined in the previous section to form $\mathrm{Au}$ seeds $\left(\left[\mathrm{HAuCl}_{4}\right]\right.$ $=0.25 \mathrm{mM},[$ citrate $]=2.5 \mathrm{mM}$, temperature of $\left.85^{\circ} \mathrm{C}\right)$ and once the seed synthesis was completed, added gold precursor $\left(\left[\mathrm{HAuCl}_{4}\right]=0.0625 \mathrm{mM}\right)$ to the solution at the temperature of $85^{\circ} \mathrm{C}$. The excess citrate present in solution from the initial seeds synthesis reduced the freshly added gold precursor during the growth step. When replicating this experiment, we observed that the SPR peak position initially red-shifted from $520 \mathrm{~nm}$ (of the seeds) moving to lower energies, leveled off at $\sim 525 \mathrm{~nm}$, and then blue-shifted to eventually level off again at $520 \mathrm{~nm}$ (Figure 6a). These findings are in agreement with the measurements performed by Polte et al., ${ }^{23}$ who observed the
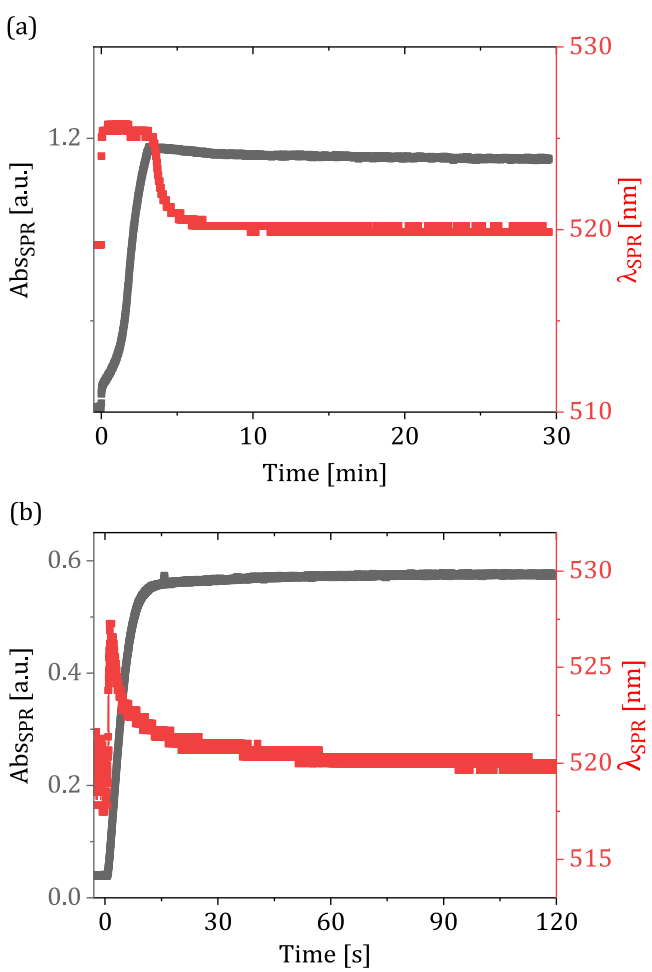

Figure 6. SPR peak absorption, $\mathrm{Abs}_{\mathrm{SPR}}$, and position, $\lambda_{\mathrm{SPR}}$, from in situ time-resolved UV-vis spectra of (a) the "citrate growth" experiment performed in this work (8.9 nm radius seeds with an $\mathrm{Au}(0)$ concentration of $0.25 \mathrm{mM}$ produced at $85^{\circ} \mathrm{C}$ with $2.5 \mathrm{mM}$ of citrate, and grown with the addition of gold precursor, final concentration of $0.0625 \mathrm{mM}$ ) and (b) the "hydroxylamine growth" experiment performed in this work $(6.3 \mathrm{~nm}$ radius seeds with an $\mathrm{Au}(0)$ concentration of $0.03 \mathrm{mM}$, grown with the addition of hydroxylamine and gold precursor, final concentrations of $0.93 \mathrm{mM}$ and $0.1 \mathrm{mM}$, respectively).

same initial red-shift followed by a progressive blue-shift in the SPR position.

We next performed a different seeded-growth experiment, starting from preformed seeds of $6.25 \mathrm{~nm}$ radius produced according to Panariello et al. ${ }^{34}$ (see Supporting Information, Section S6), and grew them via "hydroxylamine growth". ${ }^{32}$ In this synthesis, the reduction of the gold precursor was carried out by hydroxylamine, which can only reduce the gold precursor in the presence of a gold surface. ${ }^{32}$ Despite the different synthetic route, the spectra showed similar features in terms of evolution of the SPR peak position (Figure 6b), further supporting the conclusion that gold precursor species interact with the surface of the preformed particles, causing the red-shift of the colloid spectra.

The spectra acquired during the seeded-growth experiments were fitted with the same model employed in the seedless Turkevich synthesis, returning the fitting parameters $R_{c}, R_{s}, N$, $g_{s}$, and $A_{s}^{*}$. The profiles of particle size and concentration for both experiments, obtained from the spectra fitting, as well as the temporal evolution of SPR peak position and absorbance, are reported in Figure 7 and 8. Both seeded-growth experiments fitting revealed a final size in good agreement with TEM measurements (see Supporting Information, Sections S4 and S6). Comparing the results obtained from the citrate growth spectra fitting with the SAXS measurements from Polte et al., ${ }^{23}$ we observed good quantitative agreement 
(a)

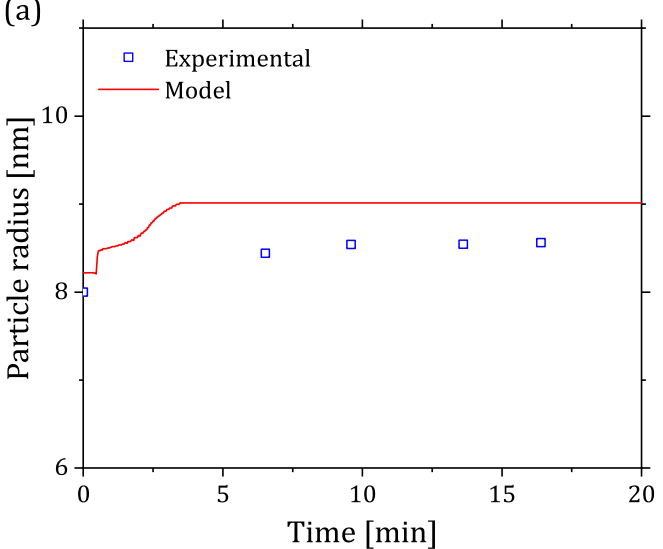

(c)

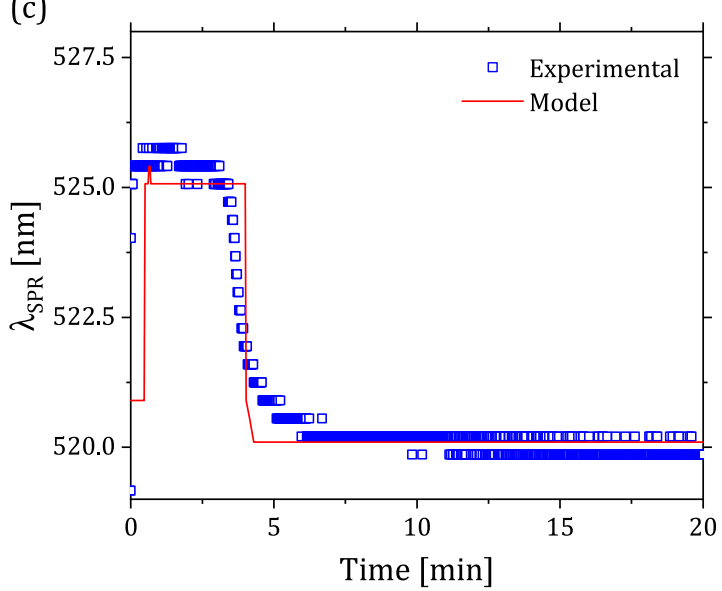

(b)

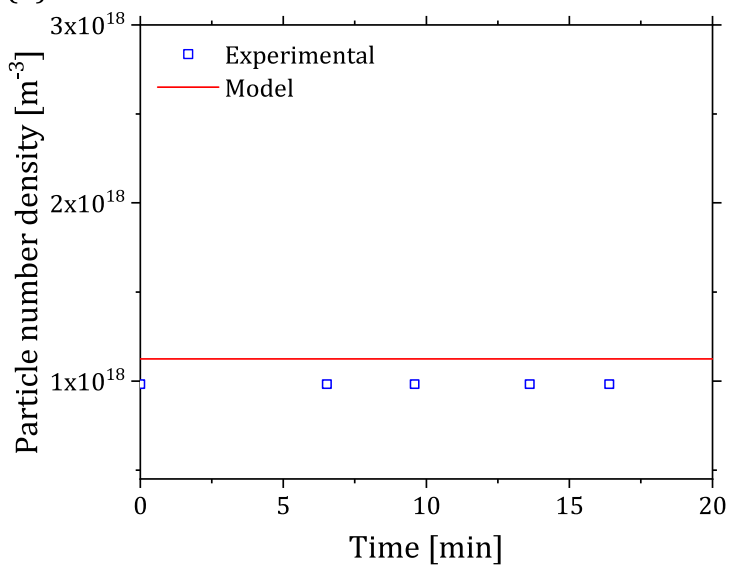

(d)

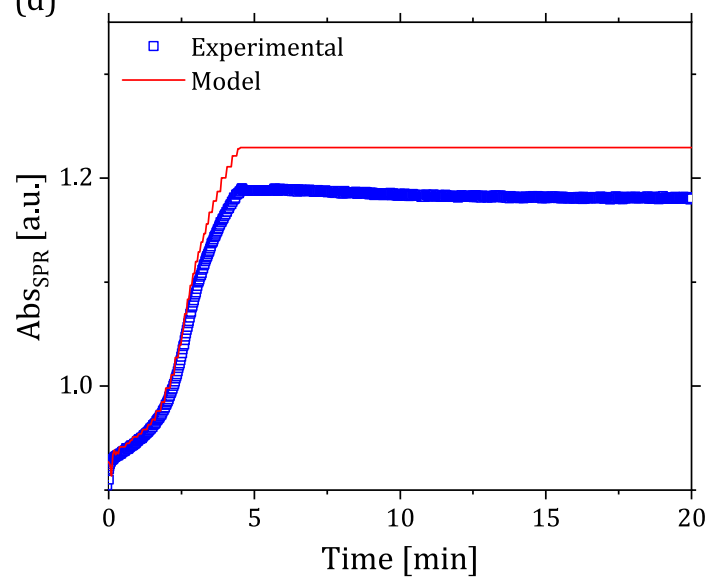

Figure 7. Evolution of particle size and number density obtained from spectra fitting of the "citrate growth" experiment and comparison between experimental and modeled peak absorbance, $\mathrm{Abs}_{\mathrm{SPR}}$ and peak position, $\lambda_{\mathrm{SPR}}$, throughout the synthesis replicating Polte et al. ${ }^{23}$ (a) Particle radius and (b) particle number density obtained from the fitting of our UV-vis spectra (red solid lines) against the data obtained in the same experiment by Polte et al. via SAXS (blue open symbols); (c) $\lambda_{\mathrm{SPR}}$ and (d) $\mathrm{Abs}_{\mathrm{SPR}}$ obtained from the modeled spectra (solid red lines) against the corresponding experimental values (open blue symbols) taken from our experiments. Evolution of the shell thickness $R_{s}$, the shell conduction electron damping frequency correction factor $A_{s}^{*}$, and the shell electron density scaling factor $g_{s}$ during the synthesis are reported in Figure S5a.

for the evolution of the particle size and particle number concentration, supporting the validity of our model.

One can observe that the fitting provides a similar shell thickness as the one obtained when fitting the spectra of the Turkevich synthesis (Figure 5a): $0.5 \mathrm{~nm}$ in the Turkevich synthesis against $0.23 \mathrm{~nm}$ (Figure S5a) in the "citrate growth" experiment and $0.5 \mathrm{~nm}$ in the "hydroxylamine growth" experiment (Figure S5b). The small difference of shell thickness observed in the experiments could be explained as follows: in the seedless Turkevich synthesis, "bare" Au particles are initially formed in the early stage of the synthesis, rapidly coated by Au precursor species, due to their higher affinity to the particle surface as compared to citrate. Citrate only starts adsorbing in large quantity on the surface of the particles when most of the precursor has already been converted to $\mathrm{Au}(0)$, when the shell thickness in Figure 5a starts decreasing, $\sim 25$ min after the beginning of the synthesis. In the "citrate growth", the seeds used are coated with citrate, and the amount of $\mathrm{Au}$ (III) added is almost 2 orders of magnitude lower than that of the excess citrate present in solution $(0.0625$ $\mathrm{mM}$ of $\mathrm{Au}(\mathrm{III})$ after addition against $\sim 2.5 \mathrm{mM}$ of citrate in solution, with an $\mathrm{Au}(\mathrm{III}) /$ citrate ratio of 40 , against a ratio of 10 used in the seedless Turkevich synthesis). It is reasonable to conclude that during the "citrate growth" the coating of the particles is a mixture of citrate and $\mathrm{Au}(\mathrm{III})$ species, influencing the value of the shell thickness, which appears smaller than that observed in the seedless Turkevich synthesis $(0.5 \mathrm{~nm}$ against $0.23 \mathrm{~nm}$ ). In the "hydroxylamine growth" case, both the $\mathrm{Au}$ seeds and the excess citrate present in solution are significantly more diluted than in the "citrate growth" (by a factor of $\sim 10$ ). Furthermore, the amount of $\mathrm{Au}(\mathrm{III})$ added is considerably larger than that used in the "citrate growth" $(0.1 \mathrm{mM}$ against $0.0625 \mathrm{mM}$ ). This suggests that in the "hydroxylamine growth" experiment, the Au nanoparticles initially get mainly coated by $\mathrm{Au}$ (III) species, similarly to what happens in the initial stage of the seedless Turkevich synthesis, hence the similar values of shell thickness (in both cases $0.5 \mathrm{~nm}$ ), which are higher than those obtained in the "citrate growth" experiment.

The fitting returned a value of $A_{s}^{*}$ constant and equal to 1 throughout the growth for both seeded-growth experiments, whereas $g_{s}$ decreases to 0.85 . This is consistent with the values of $A_{s}^{*}$ and $g_{s}$ reported for the Turkevich synthesis, where only particles smaller than $5 \mathrm{~nm}$ radius showed an increase in the value of $A_{s}^{*}$ (Figure 5).

\section{CONCLUSIONS}

This work presents a model for the interpretation of in situ time-resolved UV-vis spectroscopy data gathered during the 
(a)

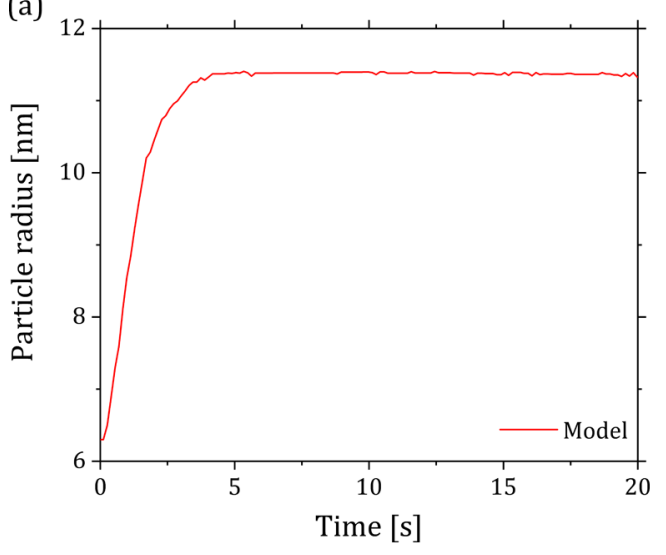

(c)

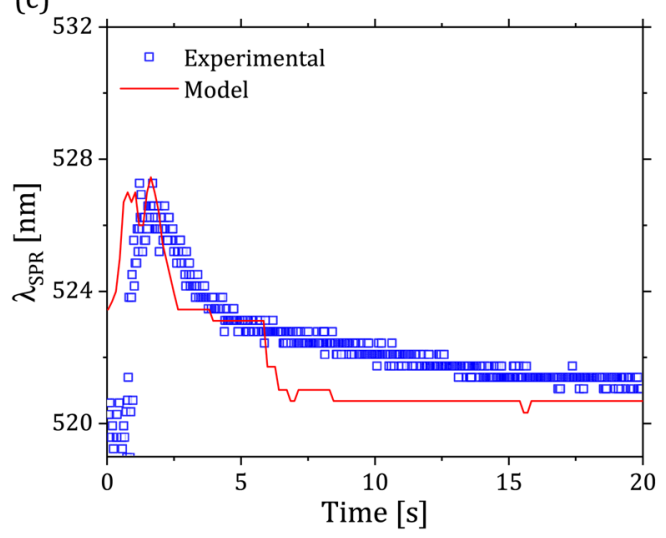

(b)

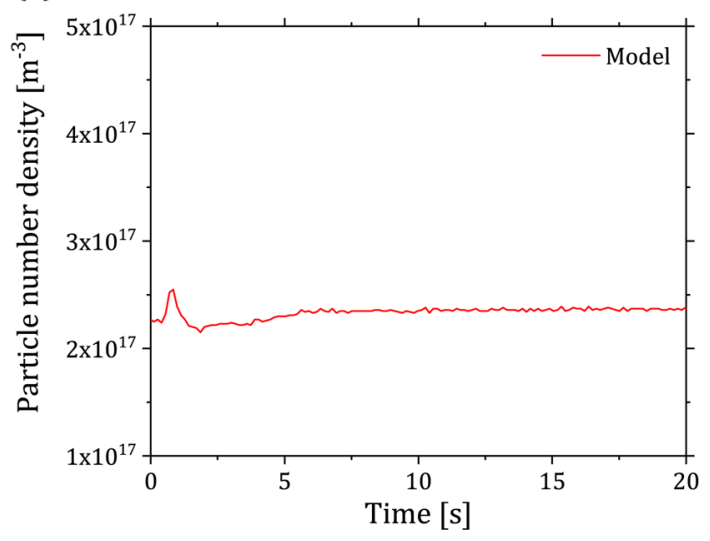

(d)

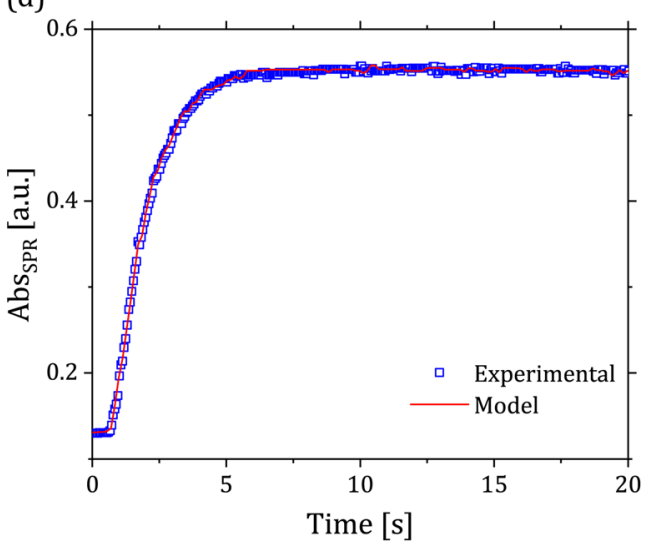

Figure 8. Evolution of particle size and number density obtained from spectra fitting of the "hydroxylamine growth" experiment and comparison between experimental and modeled peak absorbance, $\mathrm{Abs}_{\mathrm{SPR}}$ and peak position, $\lambda_{\mathrm{SPR}}$ during the synthesis. (a) Particle radius and (b) particle number density obtained from the fitting of our UV-vis spectra (solid red lines); (c) $\lambda_{\mathrm{SPR}}$ and (d) Abs $\mathrm{SPR}_{\mathrm{SP}}$ obtained from the modeled spectra (solid red lines) against the corresponding experimental values taken from our experiments (blue open symbols). Evolution during the synthesis of the shell thickness $R_{s}$, the shell conduction electron damping frequency correction factor $A_{s}^{*}$, and the shell electron density scaling factor $g_{s}$ are reported in Figure S5b.

synthesis of gold nanoparticles via the Turkevich method, returning the evolution of the main nanoparticle features during the synthesis (i.e., particle size and concentration). The spectra features observed are in line with evidence from the literature, with a progressive blue-shift in the SPR peak position during the synthesis. The spectra were fitted with a core-shell model, with a metallic outer shell with reduced conductivity and increased electron damping rate. The model describes satisfactorily the evolution of the particle size and particle number concentration when compared to SAXS measurements from the literature, as well as being able to correctly replicate the spectra features evolution during the synthesis (in terms of SPR absorbance and peak position). The lower free electron density and higher damping frequency of the outer shell are attributed to the strong interaction of the $\mathrm{Au}$ precursor species with the $\mathrm{Au}$ nanoparticle surface. We hypothesized that the precursor species, upon adsorption on the Au nanoparticle, can cause a decrease in the free electron density of the particle surface and an increase in the electron damping frequency. These two phenomena could explain the observed deviation from the behavior expected from an unperturbed gold nanoparticle. Seeded-growth experiments using preformed seeds exhibited the same SPR behavior as the conventional Turkevich synthesis. These experiments were performed using two different protocols with two distinct reducing agents (namely hydroxylamine and citrate). Despite the different protocols used, we observed the same features in the spectra as displayed by the Turkevich synthesis, suggesting that the same phenomena take place in the three different syntheses studied. Therefore, the adsorption of precursor on the particle surface appears as a reasonable explanation of the SPR behavior during these syntheses. The developed model was able to satisfactorily fit also the seeded-growth experiments, as demonstrated by the close match of the obtained particle size and number density with the results from literature SAXS experiments.

The presented model allows to reconcile the recently suggested "seed-mediated growth" mechanism for the Turkevich synthesis and the observed UV-vis spectra, disproving the presence of large aggregates that have been invoked to explain the SPR behavior during the synthesis. We hypothesized that a major role in determining the spectra features is associated with the surface chemistry of the particles and its evolution during the synthesis. Experimental investigations providing a definitive picture of the changes of the particle surface during the Turkevich synthesis are not available to the best of our knowledge. Such investigations are very challenging, particularly considering that techniques commonly employed for this purpose, such as electron microscopy, are rather invasive and can induce artifacts when performed on unstable specimens 
(such as evolving particles). Future work addressing such issues and providing a clearer description of the surface phenomena taking place during the Turkevich synthesis (and in general, during nanoparticle syntheses) will support the development and refinement of models such as the one reported here.

Retrieving features such as particle size and concentration directly from the UV-vis spectra is an extremely challenging task, as Bohren and Huffman state in their classic book on absorption and scattering of small particles: "This is the hard problem; it consists of describing a dragon from an examination of its tracks". ${ }^{48}$ We demonstrated here that the combination of UV-vis spectroscopy with other techniques providing a priori mechanistic knowledge of the synthesis (e.g., SAXS) represents a tool to gather quantitative kinetic data during nanoparticle formation. This approach can ultimately contribute to the development of quantitative kinetic models describing nanomaterial synthesis.

\section{ACKNOWLEDGMENTS}

L.P. received funding from the European Union's Horizon 2020 research and innovation programme under the Marie Skłodowska-Curie Grant Agreement No. 721290. This publication reflects only the authors' view, exempting the Community from any liability. Project website: http://cosmicetn.eu/. We also thank EPSRC (Grant EP/L027232/1) for funding.

\section{REFERENCES}

(1) Hu, M.; Chen, J.; Li, Z. Y.; Au, L.; Hartland, G. V.; Li, X.; Marquez, M.; Xia, Y. Gold Nanostructures: Engineering Their Plasmonic Properties for Biomedical Applications. Chem. Soc. Rev. 2006, 35 (11), 1084-1094.

(2) Eustis, S.; El-Sayed, M. A. Why Gold Nanoparticles Are More Precious than Pretty Gold: Noble Metal Surface Plasmon Resonance and Its Enhancement of the Radiative and Nonradiative Properties of Nanocrystals of Different Shapes. Chem. Soc. Rev. 2006, 35 (3), 209217.

(3) Gerard, D.; Gray, S. K. Aluminium Plasmonics. J. Phys. D: Appl. Phys. 2015, 48 (18), 184001.

(4) Biggins, J. S.; Yazdi, S.; Ringe, E. Magnesium Nanoparticle Plasmonics. Nano Lett. 2018, 18 (6), 3752-3758.

(5) Naik, G. V.; Shalaev, V. M.; Boltasseva, A. Alternative Plasmonic Materials: Beyond Gold and Silver. Adv. Mater. 2013, 25 (24), 32643294.

(6) Sun, Y.; Xia, Y. Shape-Controlled Synthesis of Gold and Silver Nanoparticles. Science 2002, 298, 2176-2179.

(7) Liz-Marzán, L. M. Tailoring Surface Plasmons through the Morphology and Assembly of Metal Nanoparticles. Langmuir 2006, 22 (1), 32-41.

(8) Daniel, M. C. M.; Astruc, D. Gold Nanoparticles: Assembly, Supramolecular Chemistry, Quantum-Size Related Properties and Applications toward Biology, Catalysis and Nanotechnology. Chem. Rev. 2004, 104 (1), 293-346.

(9) Huang, X.; Jain, P. K.; El-Sayed, I. H.; El-Sayed, M. A. Gold Nanoparticles: Interesting Optical Properties and Recent Applications in Cancer Diagostic and Therapy. Nanomedicine 2007, 2 (5), 681693.

(10) Pan, Y.; Neuss, S.; Leifert, A.; Fischler, M.; Wen, F.; Simon, U.; Schmid, G.; Brandau, W.; Jahnen-Dechent, W. Size-Dependent Cytotoxicity of Gold Nanoparticles. Small 2007, 3 (11), 1941-1949.

(11) Turkevich, J.; Stevenson, P. C.; Hillier, J. A Study of the Nucleation and Growth Processes in the Synthesis of Colloidal Gold. Discuss. Faraday Soc. 1951, 11, 55-75.

(12) Frens, G. Controlled Nucleation for the Regulation of the Particle Size in Monodisperse Gold Suspensions. Nature, Phys. Sci. 1973, 241, 20-22.

(13) Ji, X.; Song, X.; Li, J.; Bai, Y.; Yang, W.; Peng, X. Size Control of Gold Nanocrystals in Citrate Reduction: The Third Role of Citrate. J. Am. Chem. Soc. 2007, 129 (45), 13939-13948.

(14) Chakraborty, A.; Chakraborty, S.; Chaudhuri, B.; Bhattacharjee, $S$. Process Engineering Studies on Gold Nanoparticle Formation via Dynamic Spectroscopic Approach. Gold Bull. 2016, 49 (3-4), 75-85.

(15) Haiss, W.; Thanh, N. T. K.; Aveyard, J.; Fernig, D. G. Determination of Size and Concentration of Gold Nanoparticles from UV - Vis Spectra Determination of Size and Concentration of Gold Nanoparticles from UV - Vis Spectra. Anal. Chem. 2007, 79, 42154221.

(16) Chow, M. K.; Zukoski, C. F. Gold Sol Formation Mechanisms: Role of Colloidal Stability. J. Colloid Interface Sci. 1994, 165, 97-109.

(17) Pong, B. K.; Elim, H. I.; Chong, J. X.; Ji, W.; Trout, B. L.; Lee, J. Y. New Insights on the Nanoparticle Growth Mechanism in the Citrate Reduction of Gold(III) Salt: Formation of the Au Nanowire Intermediate and Its Nonlinear Optical Properties. J. Phys. Chem. C 2007, 111 (17), 6281-6287.

(18) Pei, L.; Mori, K.; Adachi, M. Formation Process of TwoDimensional Networked Gold Nanowires by Citrate Reduction of $\mathrm{AuCl} 4$ - and the Shape Stabilization. Langmuir 2004, 20 (18), 78377843.

(19) Wuithschick, M.; Birnbaum, A.; Witte, S.; Sztucki, M.; Vainio, U.; Pinna, N.; Rademann, K.; Emmerling, F.; Kraehnert, R.; Polte, J. 
Turkevich in New Robes: Key Questions Answered for the Most Common Gold Nanoparticle Synthesis. ACS Nano 2015, 9 (7), $7052-7071$.

(20) Polte, J.; Ahner, T. T.; Delissen, F.; Sokolov, S.; Emmerling, F.; Thünemann, A. F.; Kraehnert, R. Mechanism of Gold Nanoparticle Formation in the Classical Citrate Synthesis Method Derived from Coupled In Situ XANES and SAXS Evaluation. J. Am. Chem. Soc. 2010, 132 (9), 1296-1301.

(21) Wuithschick, M.; Birnbaum, A.; Witte, S.; Sztucki, M.; Vainio, U.; Pinna, N.; Rademann, K.; Emmerling, F.; Kraehnert, R.; Polte, J. Turkevich in New Robes: Supporting Information. ACS Nano 2015, 9 (7), 7052-7071.

(22) Kettemann, F.; Birnbaum, A.; Witte, S.; Wuithschick, M.; Pinna, N.; Kraehnert, R.; Rademann, K.; Polte, J. Missing Piece of the Mechanism of the Turkevich Method: The Critical Role of Citrate Protonation. Chem. Mater. 2016, 28 (11), 4072-4081.

(23) Polte, J.; Herder, M.; Erler, R.; Rolf, S.; Fischer, A.; Würth, C.; Thünemann, A. F.; Kraehnert, R.; Emmerling, F. Mechanistic Insights into Seeded Growth Processes of Gold Nanoparticles. Nanoscale 2010, 2 (11), 2463-2469.

(24) Polte, J.; Erler, R.; Thünemann, A. F.; Emmerling, F.; Kraehnert, R. SAXS in Combination with a Free Liquid Jet for Improved Time-Resolved in Situ Studies of the Nucleation and Growth of Nanoparticles. Chem. Commun. 2010, 46 (48), 9209.

(25) Agunloye, E.; Panariello, L.; Gavriilidis, A.; Mazzei, L. A Model for the Formation of Gold Nanoparticles in the Citrate Synthesis Method. Chem. Eng. Sci. 2018, 191, 318-331.

(26) Kumar, S.; Gandhi, K. S.; Kumar, R. Modeling of Formation of Gold Nanoparticles by Citrate Method. Ind. Eng. Chem. Res. 2007, 46 (10), 3128-3136.

(27) Agunloye, E.; Gavriilidis, A.; Mazzei, L. A Mathematical Investigation of the Turkevich Organizer Theory in the Citrate Method for the Synthesis of Gold Nanoparticles. Chem. Eng. Sci. 2017, 173, 275-286.

(28) Mulvaney, P.; Linnert, T.; Henglein, A. Surface Chemistry of Colloidal Silver in Aqueous Solution: Observations on Chemisorption and Reactivity. J. Phys. Chem. 1991, 95, 7843-7846.

(29) Berchmans, S.; Thomas, P. J.; Rao, C. N. R. Novel Effects of Metal Ion Chelation on the Properties of Lipoic Acid-Capped Ag and Au Nanoparticles. J. Phys. Chem. B 2002, 106 (18), 4647-4651.

(30) Peng, S.; McMahon, J. M.; Schatz, G. C.; Gray, S. K.; Sun, Y. Reversing the Size-Dependence of Surface Plasmon Resonances. Proc. Natl. Acad. Sci. U. S. A. 2010, 107 (33), 14530-14534.

(31) Averitt, R. D.; Sarkar, D.; Halas, N. J. Plasmon Resonance Shifts of $\mathrm{Au}$-Coated $\mathrm{Au}_{2} \mathrm{~S}$ Nanoshells: Insight into Multicomponent Nanoparticle Growth. Phys. Rev. Lett. 1997, 78 (22), 4217-4220.

(32) Brown, K. R.; Natan, M. J. Hydroxylamine Seeding of Colloidal Au Nanoparticles in Solution and on Surfaces. Langmuir 1998, 14 (4), $726-728$.

(33) Mondini, S.; Ferretti, A. M.; Puglisi, A.; Ponti, A. Pebbles and PebbleJuggler: Software for Accurate, Unbiased, and Fast Measurement and Analysis of Nanoparticle Morphology from Transmission Electron Microscopy (TEM) Micrographs. Nanoscale 2012, 4 (17), $5356-5372$.

(34) Panariello, L.; Damilos, S.; du Toit, H.; Wu, G.; Radhakrishnan, A. N. P.; Parkin, I. P.; Gavriilidis, A. Highly Reproducible, High-Yield Flow Synthesis of Gold Nanoparticles Based on a Rational Reactor Design Exploiting the Reduction of Passivated Au(III). React. Chem. Eng. 2020, 5, 663-676.

(35) Mulvaney, P.; Pérez-Juste, J.; Giersig, M.; Liz-Marzán, L. M.; Pecharromán, C. Drastic Surface Plasmon Mode Shifts in Gold Nanorods Due to Electron Charging. Plasmonics 2006, 1 (1), 61-66.

(36) Sun, Y.; Gray, S. K.; Peng, S. Surface Chemistry: A NonNegligible Parameter in Determining Optical Properties of Small Colloidal Metal Nanoparticles. Phys. Chem. Chem. Phys. 2011, 13 (25), 11814-11826.

(37) Myroshnychenko, V.; Rodríguez-Fernández, J.; PastorizaSantos, I.; Funston, A. M.; Novo, C.; Mulvaney, P.; Liz-Marzán, L.
M.; García de Abajo, F. J. Modelling the Optical Response of Gold Nanoparticles. Chem. Soc. Rev. 2008, 37 (9), 1792-1805.

(38) Kreibig, U.; Genzel, L. Optical Absorption of Small Metallic Particles. Surf. Sci. 1985, 156, 678-700.

(39) Mulvaney, P. Surface Plasmon Spectroscopy of Nanosized Metal Particles. Langmuir 1996, 12 (3), 788-800.

(40) Amendola, V.; Meneghetti, M. Size Evaluation of Gold Nanoparticles by UV - Vis Spectroscopy. J. Phys. Chem. C 2009, 113 (11), 4277-4285.

(41) Amendola, V.; Pilot, R.; Frasconi, M.; Maragò, O. M.; Iati, M. A. Surface Plasmon Resonance in Gold Nanoparticles: A Review. J. Phys.: Condens. Matter 2017, 29, 203002.

(42) Biggs, S.; Chow, M. K.; Zukoski, C. F.; Grieser, F. The Role of Colloidal Stability in the Formation of Gold Sols. J. Colloid Interface Sci. 1993, 160, 511-513.

(43) Hendel, T.; Wuithschick, M.; Kettemann, F.; Birnbaum, A.; Rademann, K.; Polte, J. In Situ Determination of Colloidal Gold Concentrations with UV-Vis Spectroscopy: Limitations and Perspectives. Anal. Chem. 2014, 86 (22), 11115-11124.

(44) Ojea-Jiménez, I.; Campanera, J. M. Molecular Modeling of the Reduction Mechanism in the Citrate-Mediated Synthesis of Gold Nanoparticles. J. Phys. Chem. C 2012, 116 (44), 23682-23691.

(45) Gao, Y.; Torrente-Murciano, L. Mechanistic Insights of the Reduction of Gold Salts in the Turkevich Protocol. Nanoscale 2020, 12 (4), 2740-2751.

(46) Novo, C.; Funston, A. M.; Mulvaney, P. Direct Observation of Chemical Reactions on Single Gold Nanocrystals Using Surface Plasmon Spectroscopy. Nat. Nanotechnol. 2008, 3, 598-602.

(47) Persson, B. N. J. Polarizability of Small Spherical Metal Particles: Influence of the Matrix Environment. Surf. Sci. 1993, 281 (1-2), 153-162.

(48) Bohren, C. F.; Huffman, H. R. Absorption and Scattering of Light by Small Particles; Wiley\&Sons: Hoboken, NJ, 1983. 